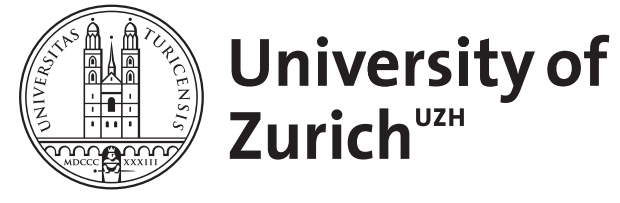
Archive

University of Zurich

University Library

Strickhofstrasse 39

CH-8057 Zurich

www.zora.uzh.ch

Year: 2021

\title{
Am Fluchtpunkt der Fotografie
}

\author{
Laleg, Dominique
}

DOI: https://doi.org/10.1515/zkg-2021-2008

Posted at the Zurich Open Repository and Archive, University of Zurich

ZORA URL: https://doi.org/10.5167/uzh-205259

Journal Article

Published Version

Originally published at:

Laleg, Dominique (2021). Am Fluchtpunkt der Fotografie. Zeitschrift für Kunstgeschichte, 84(2):281-286.

DOI: https://doi.org/10.1515/zkg-2021-2008 


\section{Am Fluchtpunkt der Fotografie}

Michalis Valaouris, Perspektive und Fotografie: Studien zur Naturalisierung des Kamerabildes (Kaleidogramme, Bd. 174)

Berlin: Kulturverlag Kadmos, 2018, 269 Seiten, 53 S/W-Abbildungen, € 24,90,

ISBN 978-3-86599-414-1

Rezensiert von Dominique Laleg

Bildnerische Mittel sind nicht bloß technische Verfahren zur Bilderzeugung, sondern bilden darüber hinaus Komplexe, in denen Materialien, Techniken und wissenschaftliche Erkenntnisse zusammentreffen. Obschon sie als solche immer historisch sind, weisen manche bildnerische Mittel Eigenschaften auf, die sie auf erstaunlich anhaltende Weisen wirksam machen. Während diese anhaltende Wirksamkeit für die Historiker*innen üblicherweise innerhalb eines bestimmten Kontextes steht, sind die Theoretiker*innen meist auf der Suche nach einer gleichsam übergeordneten Logik, deren Historizität zur Debatte steht. Michalis Valaouris hat eine Untersuchung vorgelegt, die es sich zur Aufgabe macht, diesem doppelten Anspruch von Geschichtsschreibung einerseits und Theorie andererseits gerecht zu werden. Das bildnerische Mittel, das er untersucht, hat denn auch Historiker*innen und Theoretiker*innen gleichermaßen beschäftigt: die Linear- oder Fluchtpunktperspektive. Dabei geht es Valaouris nicht darum, die umfangreiche Literatur zur Perspektive um eine weitere Arabeske zu ergänzen, sondern er zielt auf eine geradezu auffällige Lücke in der Forschung, die sich zwischen zwei großen Gebieten auftut: zwischen der Fototheorie und der Perspektivtheorie. Diese Lücke erstaunt vor allem deshalb, weil namhafte Autor*innen aus beiden Gebieten den offensichtlichen Zusammenhang von Zentralperspektive und Fotografie schon seit rund einhundert
Jahren erwähnen, allerdings ohne diesen jemals systematisch zu untersuchen. Die Unklarheit der Randbemerkungen von Autoren wie Erwin Panofsky, Hubert Damisch, Pierre Bourdieu oder Roland Barthes zum Zusammenhang von Perspektive und Fotografie nimmt Valaouris zum Anlass seiner Untersuchung. Wie genau ist der Zusammenhang zu verstehen zwischen der Perspektive als einer ursprünglich geometrischen Zeichentechnik des 15. Jahrhunderts und der Fotografie als automatisierter Bildproduktion, die erst im 19. Jahrhundert erfunden wurde? Dieser Frage geht der Autor in drei Kapiteln nach.

Im ersten Kapitel (Vorgeschichte der Fotografie) werden zunächst drei unterschiedliche historische Deutungen der Fotografie rekonstruiert: Heinrich Schwartz' Eingliederung der Fotografie in die Kunstgeschichte als Ergebnis wissenschaftlicher, sozialer und künstlerischer Faktoren; John Szarkowskis bzw. Peter Galassis modernistische Deutung der Fotografie als einer ästhetischen und nicht wissenschaftlich-technisch begründeten Bildpraxis; und schließlich Geoffrey Batchens poststrukturalistische Deutung der Fotografie als Medium der Differenz, das zwischen Natur und Kultur changiert. Diese Vorgeschichte(n) der Fotografie problematisiert der Autor, indem er ihre historiografischen Fallstricke verdeutlicht. Es handle sich entweder um einen anachronistischen Rückblick, der vom historischen Geburtsmoment der Fotografie im Jahr 1839 rückwärts gerichtet ist, um Phänomene auszumachen, in denen die Idee der Fotografie bereits enthalten sei. Andererseits laufe eine Vorgeschichte der Fotografie Gefahr, in historisch autonomen Phänomenen eine Teleologie zu erkennen, die in der Erfindung der Fotografie zu kulminieren scheint. Diese historiografische Problematik, ihre Anachronismen und Zirkelschlüsse bringen den Autor zum Schluss, dass kein kausales Verhältnis zwischen der Zentral- 
perspektive des 15. Jahrhunderts und der Erfindung der Fotografie im 19. Jahrhundert historisch präzisiert werden kann. Trotz der augenfälligen formalen Analogien zwischen linearperspektivischen und fotografischen Bildern seien die rund 400 Jahre, die zwischen den beiden Erfindungen liegen, ein für die strenge Geschichtsschreibung ahistorischer Zeitraum. Aus diesem Grund schlägt der Autor vor, der Problematik auf theoretischem Weg zu begegnen: Es geht ihm nicht um die historische Kausalität zwischen Perspektive und Fotografie, sondern Valaouris richtet den Fokus auf die strukturellen Beziehungen der beiden, um so das Fortleben der Perspektive im fotografischen Medium zu beschreiben.

Die zentrale Frage, die im zweiten Kapitel der Studie (Die Kamera und das Dispositiv der Perspektive) verfolgt wird, lautet: »Inwiefern lässt sich die Tradition der Perspektive in den medialen Bedingungen der Fotografie erkennen?« $(41-42)$. Dabei arbeitet der Autor die Analogien zwischen Perspektive und Fotografie systematische heraus und fokussiert dabei drei Elemente der Kamera: Die Blende, die mit Roland Barthes als sténope bezeichnet wird; das damit erzeugte Lichtstrahlenbündel im Innern der Kamera; und das Lichtbild, welches der Autor ebenfalls in Berufung auf Barthes als eidolon benennt. Gemeinsam bilden diese drei das Dispositiv, das der fotografischen Bildgebung zu Grunde liege und diese untrennbar mit der Perspektive verbinde. Denn wie Valaouris zeigt, entspricht die Dreigliedrigkeit und ihr systematisches Zusammenspiel demjenigen der Perspektive, deren Prinzip ebenfalls auf einer punktuell bezogenen Projektion von Strahlen basiert, als deren Querschnitt das perspektivische Bild konzipiert ist. Um diese "strukturelle Analogie« (64) zu verdeutlichen, bezieht sich Valaouris auf jenes Ereignis, das in der Forschung zur Perspektive vielfach als deren Ursprungsmoment diskutiert wird. ${ }^{1}$ Gemäß der schriftlichen Überlieferung Antonio Manettis hat die Zentralperspektive einen experimentellen Ursprung, nämlich die Vorrichtung, die

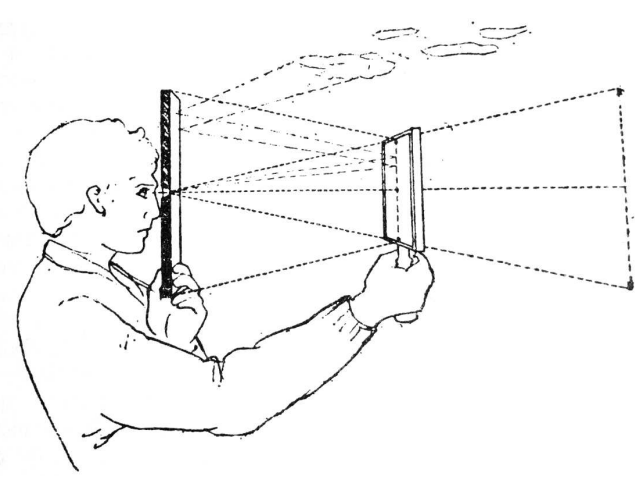

1 Rekonstruktion der ersten perspektivischen Demonstration (ca. 1420) von Filippo Brunelleschi in Florenz nach Alessandro Parronchi, 1964

Filippo Brunelleschi gebrauchte, als er um das Jahr 1420 auf dem Domplatz von Florenz seine perspektivische Darstellung des Baptisteriums überprüfte. Die kleine Bildtafel, die am Fluchtpunkt mit einem Loch versehen war, durch das mit einem Auge hindurchgesehen werden konnte, um die Spiegelung derselben Tafel und des Blicks in einem frontal positionierten Spiegel zu sehen (Abb. 1), bildet in diesem Sinn auch den indirekten Ursprung der Fotografie. Denn das kleine Loch in Brunelleschis Tafel ist zugleich Fluchtpunkt und Augenpunkt und entspreche daher der Blende der Kamera als einer sténope, d.h. jenem kleinen Loch, welches das Zentrum des Strahlenbündels bildet, wodurch das Lichtbild auf die Projektionsfläche geworfen wird. In Berufung auf Hubert Damischs monumentale Studie Der Ursprung der Perspektive versteht der Autor das Zusammenspiel dieser drei Momente als »regulierendes Dispositiv« (54), das in der Kamera am Werk ist. Das philosophische Gewicht, das sich der Autor mit seinem Gewährsmann Damisch einholt, könnte kaum größer sein, versteht Damisch die Perspektive doch als theoretisches Objekt, das nicht nur die Konstitution von Subjektivität leistet, sondern diese Subjektgenese für das sehende Subjekt zugleich erlebbar macht und so für deren fundamentale Reflexion sorgt. Weiter deutet Damischs strukturalistisch 
geprägte Interpretation die Perspektive zugleich als ein sprachähnliches Dispositiv, das gleichsam eine visuelle Grammatik ermöglicht. Die frontale Korrelation von Augpunkt und Fluchtpunkt vergleicht er mit den Ortsadverbien (hier-dort) sowie den Personalpronomina (ich-du). Zugleich begreift Damisch die Ordnung der Tiefenfluchten der Darstellung, die auf den Fluchtpunkt zulaufen, als Deklinationen, insofern sie eine formale Flexion bedeuten und damit der grammatischen Beugung gleichen. Diese grammatische Dimension der Perspektive erwähnt Valaouris zwar kurz (54), doch stehen für ihn die subjekttheoretischen Argumente Damischs im Vordergrund. Es ist die Subjektivität als Effekt des Dispositivs, die gleichsam in die Kamera implementiert ist, welche nicht nur perspektivische Bilder produziere, sondern auch »den >Blick (70). Die Perspektive wirkt in der Fotografie also in einem zweifachen Sinn: Erstens strukturell in der Disposition von sténope, Lichtstrahlenbündel und eidolon, deren Zusammenspiel dann zweitens eine "symbolische Funktion" (70) erfüllt, welche den subjektiven Blick in das fotografische Bild einschreibt. Die Analogie, um die sich Valaouris' Studie insgesamt dreht, betrifft also nicht nur die Struktur, sondern auch den Effekt, den Kameraprojektion und perspektivische Geometrie teilen. Der Autor führt so die subjekttheoretische Tragweite der Perspektivforschung in den Diskurs zur Fotografie ein. Dies sei deshalb dringend nötig, weil sich die Fototheorie seit den 1980er Jahren einseitig auf den Aspekt der Indexikalität des Fotografischen konzentriert habe. In diesem Sinn paradigmatisch sei die Fototheorie von Roland Barthes, die den Fokus ganz auf die fotografische Spur legt und sich explizit von einer Bestimmung der Fotografie als genuin perspektivischem Medium distanziert. Diese verbreitete Konzeption der Fotografie als einem wesentlich indexikalischen Bildgebungsverfahren will die Studie nicht etwa überwinden oder ersetzen, sondern ergänzen. Denn dem mittels gebündelter Lichtstrahlen erzeugten Lichtbild (eidolon), das sich als Spur im Film einbrennt, gehe die perspektivische Disposition der Projektion voraus. Der Index, der auf der Projektionsfläche fotochemisch fixiert wird, ist in diesem Sinn bereits perspektivisch präfiguriert, was jedoch leicht zu übersehen sei, weil die perspektivische Projektion im abgeschlossenen Gehäuse der Kamera verborgen ist. Diese technisch notwendige Verborgenheit des Produktionsprozesses ermögliche einerseits die ideologische Verfassung der Fotografie, welche wesentlich in ihrer Naturalisierung liege, andererseits rühre daher auch ihr magischer Charakter, der bereits der camera obscura und ihren bewegten Lichtbilder zugesprochen wurde (110-111). Das zweite Kapitel des Buches schließt denn auch mit den ideologiekritischen Beobachtung, dass die verborgene Implementierung der Perspektive in die Kamera den bis heute anhaltenden Glauben an die Natürlichkeit und Objektivität des Kamerabildes ermöglicht habe. Dagegen betont Valaouris, dass die perspektivische Disposition der Fotografie und ihre symbolische Operation eine genuin kulturelle (westeuropäische) Prägung aufweise, die ihrer vermeintlichen Neutralität im Grundsatz widerspreche.

Im dritten und letzten Kapitel (Fotografie und das Dispositiv der Perspektive) zieht Valaouris Bildbeispiele aus Kunst und Fotografiegeschichte heran, um seine Überlegungen an konkreten Phänomenen zu erläutern und zu vertiefen. Er zeigt, wie etwa die perspektivische Disposition der Fotografie in Werken der Gegenwartskunst reflektiert und offengelegt wird, und er erkennt darin jenen ideologiekritischen Anspruch, den seine Studie selbst formuliert. Weiter führt Valaouris mittels genauer Werkanalysen berühmter Fotografien wie Eugène Atgets Am Quai du Pont Neuf oder Gustave Le Grays Die Sonne am Zenit vor, auf welche Weise sich die Perspektive im Kamerabild konkret manifestiert und wie diese Manifestation von den Werken mal explizit und mal implizit artikuliert wird. Das Kapitel 


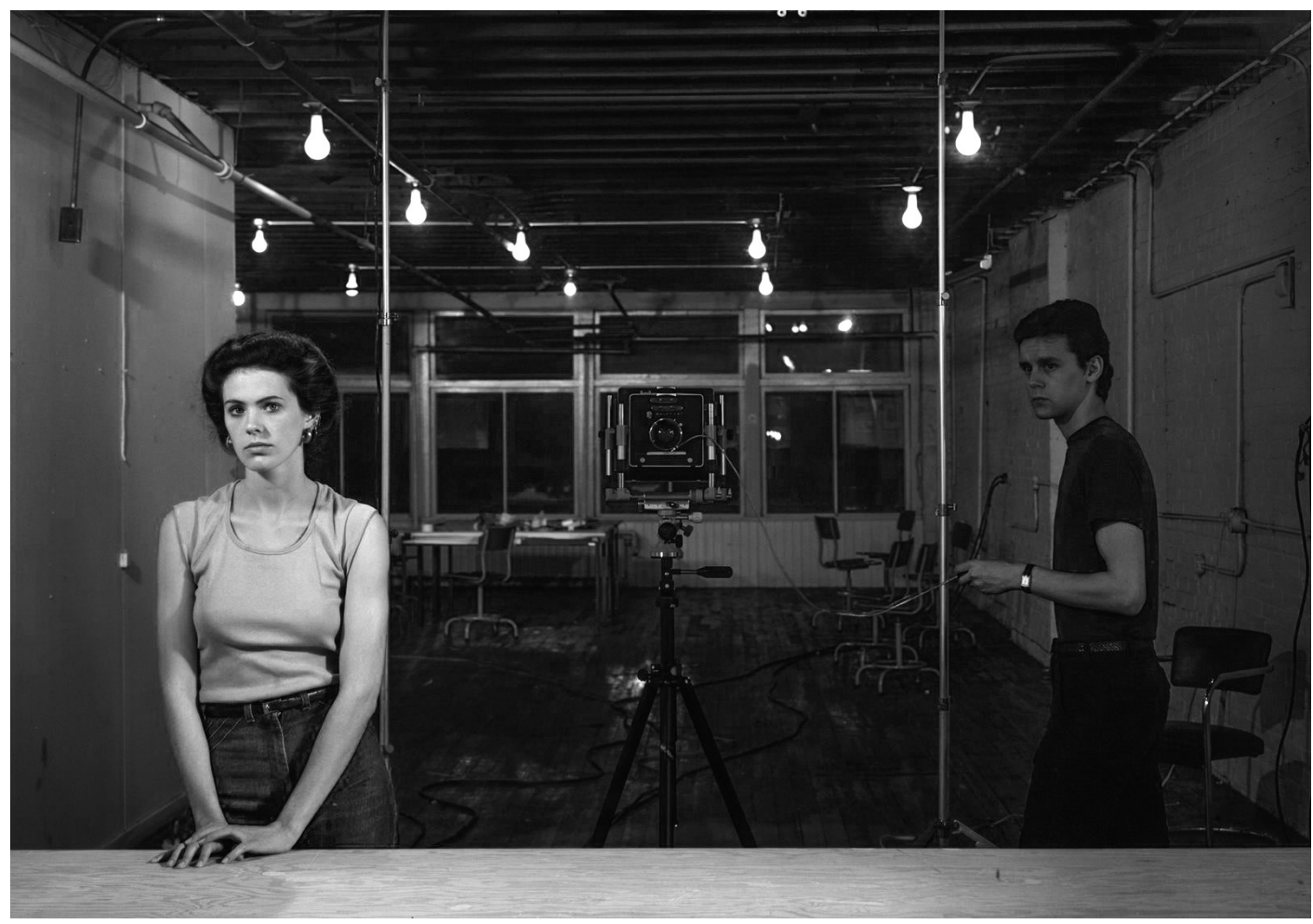

2 Jeff Wall, Picture for Women, 1979, farbige Großbilddias in Leuchtkasten, $161,5 \times 223 \times 28,5 \mathrm{~cm}$. Paris, Centre national d'art et de culture Georges Pompidou

schließt mit einer erhellenden Erläuterung dreier wenig populärer fotografischer Verfahren, die nicht dem perspektivischen Dispositiv entsprechen, der Panoramafotografie, dem Fotogramm und dem Cliché-verre. Die Besprechung dieser Nischen fotografischer Praxis ist deshalb von Interesse, weil gerade ihre Abweichungen von der dominanten Konvention die arbiträre Verfasstheit und Definition des fotografischen Mediums sichtbar machen. Das Cliché-verre etwa, bei dem die Darstellung von Hand auf eine behandelte Glasplatte gezeichnet wird, die dann, als Negativ genutzt, auf lichtempfindlichem $\mathrm{Pa}$ pier abgezogen wird, bildet eine »gemischte $\mathrm{Me}$ dialität« (205), in der sich Handzeichnung und fotografische Technik verbinden. Die Tatsache, dass Cliché-verre-Arbeiten nicht in Fotosammlungen, sondern in Kupferstichkabinetten auf- bewahrt werden, untermauert die These des Autors, dass die perspektivische Disposition des fotografischen Index unbewusst zum Medium der Fotografie gezählt wird.

Valaouris' Studie ist eine zweifach orientierte Antwort auf die lange Zeit offen gelassene Frage nach dem genauen Zusammenhang zwischen Perspektive und Fotografie. Sie leistet einerseits einen soliden Beitrag zur Theorie und Geschichte der Fotografie, indem sie die Bedeutung der Perspektive für das Kamerabild auf mehreren Ebenen untersucht, nämlich als technische Voraussetzung der fotografischen Indexikalität sowie als symbolische Funktion für Subjektivität. Die Ergänzung der Fototheorien - allen voran jene Roland Barthes' - durch die subjekttheoretischen Überlegungen der Perspektivforschung ist ein strategisch wie methodisch kluger 
Zug, da er zugleich einen Beitrag zur Perspektivtheorie leistet. Zwar verwenden Theoretiker wie Samuel Edgerton und Hubert Damisch in ihren Studien eine ganze Reihe von Fotografien, ${ }^{2}$ um den experimentellen Ursprung der Perspektive $\mathrm{zu}$ analysieren, doch ohne auf die Frage einzugehen, welche Voraussetzungen der Kamera diese fotografische Untersuchungsmethode überhaupt möglich macht. Genau diese Voraussetzungen untersucht die Studie in einer gleichsam non-foucaultschen Weise. Denn anders als etwa Jonathan Crary in seiner berühmten Studie zu den Wahrnehmungsdispositiven des 19. Jahrhunderts ${ }^{3}$ fokussiert Valaouris gerade nicht historische Brüche, sondern die Kontinuität in der Geschichte der Perspektive. Den Preis, den er für diesen Fokus bezahlt, äußert sich darin, dass die Analogie von Perspektive und Fotografie gelegentlich feine Unterschiede übersieht, die für die Frage der spezifischen Wirksamkeit des perspektivischen Dispositivs in der Kamera aufschlussreich wären. Wenn etwa der SubjektEffekt der Apparatur Brunelleschis auch für die Kamera geltend gemacht wird, unterschlägt der Autor die frappanten Unterschiede zwischen den beiden Dispositionen. Während Brunelleschis Blick in seinen Prototyp, wie Damisch sagt, in einem geschlossenen Spiegelkreislauf gefangen ist und daher zu Recht als "monadisch» begriffen werden kann, ist der Blick durch die Kamera immer durchlässig auf die Welt. Anders als beim außergewöhnlichen Beispiel von Jeff Walls Picture for Women (Abb. 2) wirft normalerweise kein Spiegel den Blick des Fotografen direkt auf sein Auge zurück, oder aber höchstens - wie bei der Spiegelreflexkamera - für den Sekundenbruchteil der Belichtung, wenn nämlich der Spiegel, der das Lichtstrahlenbündel zum Sucher umlenkt, kurz hochgeklappt ist. Derartige Modifikationen des perspektivischen Dispositivs in der Kamera sind deshalb relevant, weil damit der für die Fotografie zentrale Aspekt der Zeitlichkeit eingeführt wird, welcher später etwa - wenn auch auf andere Weise - den mehrfach zitierten
Filmtheoretiker Jean-Louis Baudry beschäftigen wird. Da die Studie von Valaouris jedoch stets auf den kleinsten gemeinsamen Nenner von Perspektive und Fotografie konzentriert ist (sténope, Lichtstrahlenbündel und eidolon), entgehen ihr gelegentlich solche technisch und historisch wichtigen Veränderungen sowie ihre subjekttheoretischen Konsequenzen.

Bedauerlich ist auch, dass neuere Entwicklungen in der fotografischen Technik und Praxis fast vollständig ausgespart werden. Nur in einem kurzen Ausblick am Ende des Textes formuliert der Autor die Frage nach dem Fortleben des perspektivischen Dispositivs in der digitalen Technologie, delegiert die Beantwortung jedoch an die weitere Forschung. Die in diesem Sinn konsequent historische Ausrichtung der Studie läuft so Gefahr, mit den eigenen Überlegungen in der untersuchten Geschichte haften zu bleiben. Das äußert sich etwa im stellenweise ausgeprägt ideologiekritischen Anspruch, der bereits im Untertitel veranschlagt wird. Zwar zeigt die Studie schlüssig auf, dass die Naturalisierung des Kamerabildes im 19. und 20. Jahrhundert eine wichtige Rolle für die Konzeption des fotografischen Mediums spielt, übergeht aber stillschweigend, dass die Digitalisierung der Fotografie in den vergangenen zwanzig Jahren ein breites Bewusstsein um die Artifizialität fotografischer Bilder erzeugt hat. Der Autor selbst lebt in einer Welt, in der jedes Kind auf dem Smartphone mit Bildbearbeitungssoftware jongliert und etwa Belichtung, Tonwerte und selbst perspektivische Verzerrungen des Kamerabildes in wenigen Sekunden gezielt verändern kann. Vor diesem Hintergrund erscheint der ideologiekritische Anspruch des Autors selbst historisch.

Dennoch bildet Valaouris' Studie eine profunde und elaborierte Auseinandersetzung mit einem historisch, theoretisch und ästhetisch hochrelevanten Themenkomplex. Sie überzeugt durch die historische sowie philologische Sorgfalt, mit der Quellen bearbeitet und in Beziehung gesetzt werden, sowie durch den stets in Gang 
gehaltenen Dialog zwischen Theorie und Bildbeispielen. Mit diesen gut gewählten und dabei vielseitigen Bildern gelingt es dem Autor, seine Grundthese glaubhaft darzustellen: Dass die Perspektive als historisch-kulturelles Spezifikum unter Bewahrung ihrer ursprünglichen Symbolisierungsfunktion flexibel genug ist, um in unterschiedlichsten Kontexten weiterzuwirken, und sie so das Medium Fotografie wesentlich mitkonstituiert. Die am Ende formulierte Frage nach dem globalen Erfolg der Perspektive ist zwar lediglich als Ausblick begriffen, doch ist an dieser Stelle längst klar, dass die kritisierte Naturalisierung und Universalisierung der Perspektive mit einem gleichsam pikturalen Imperialismus verbunden ist. Wäre sonst der globale Erfolg der Perspektive ohne die Fotografie überhaupt denkbar? Eine solide Grundlage für diese postkolonialistische Kritik der Perspektive hat die Studie bereits gelegt.

1 Siehe etwa James Elkins, The Poetics of Perspective, Ithaca 1994; Hubert Damisch, Der Ursprung der Perspektive, Zürich 2010.

2 Samuel Edgerton, Die Entdeckung der Perspektive, München 2002; Damisch 2010 (wie Anm. 1).

3 Jonathan Crary, Techniken des Betrachters: Sehen und Moderne im 19. Jahrhundert, Dresden/Basel 1996.

Abbildungsnachweis: 1 Aus dem besprochenen Band, 220. — 2 aus Ina Conzen (Hg.), Mythos Atelier: Von Spitzweg bis Picasso, von Giacometti bis Nauman (Ausst.-Kat. Stuttgart, Staatsgalerie), München 2012, 212. 